\title{
Chronic recurrent multifocal osteomyelitis (CRMO): evidence for a susceptibility gene located on chromosome 18q21.3-18q22
}

\author{
Astrid Golla ${ }^{\star 1,4,5}$, Annette Jansson ${ }^{2,5}$, Juliane Ramser ${ }^{1}$, Heide Hellebrand ${ }^{1}$, Robert Zahn ${ }^{3}$, \\ Thomas Meitinger ${ }^{1}$, Bernd H Belohradsky ${ }^{2}$ and Alfons Meindl ${ }^{1}$ \\ ${ }^{1}$ Abteilung Medizinische Genetik der Kinderklinik und Kinderpoliklinik im Dr. von Hauneŕschen Kinderspital, \\ Universität München, München, Germany; ${ }^{2}$ Abteilung Antimikrobielle Therapie und Infektionsimmunologie der \\ Kinderklinik und Kinderpoliklinik im Dr. von Haunerschen Kinderspital, Universität München, München, Germany; \\ ${ }^{3}$ Labor für Immungenetik der Kinderklinik und Kinderpoliklinik im Dr. von Hauneŕschen Kinderspital, Universität \\ München, München, Germany; ${ }^{4}$ Institut für Medizinische Biometrie, Informatik und Epidemiologie, Universität \\ Bonn, Bonn, Germany
}

Chronic recurrent multifocal osteomyelitis (CRMO) is characterised by recurrent inflammatory lesions in the metaphyses of long bones and usually affects children and adolescents. Similarity with an autosomal recessive mouse disorder (cmo, chronic multifocal osteomyelitis) prompted us to perform a family based association study with two markers on chromosome $\mathbf{1 8 q}$ in the region homologous to the cmo localisation of the mouse. We found a significant association of CRMO with a rare allele of marker D18560, resulting in a haplotype relative risk (HRR) of 18. This suggests the existence of a gene in this region contributing in a significant manner to the aetiology of CRMO and concomitantly demonstrates evidence for a genetic basis of CRMO for the first time. This gene is different from RANK, which is mutated in familial expansile osteolysis (FEO), but not in CRMO. Mutation screening in RANK and the genes PIGN and KIAA1468 led to detection of two variants (one in RANK and one in PIGN), which are in linkage disequilibrium with the rare D18S60 allele, but not independently associated with CRMO.

European Journal of Human Genetics (2002) 10, 217-221. DOI: 10.1038/sj/ejhg/5200789

Keywords: CRMO; chronic recurrent multifocal osteomyelitis; family based association study; D18S60; genetic basis

Introduction

Chronic recurrent multifocal osteomyelitis (CRMO [MIM 259680]) is a disease of unknown origin. It is characterised by recurrent inflammatory lesions predominantly in the metaphyses of long bones and usually affects children and

*Correspondence: Astrid Golla, Universität Bonn, Institut für Medizinische Biometrie, Informatik und Epidemiologie, Sigmund-FreudStraße 25, 53105 Bonn, Germany. Tel: +49 228287 5564;

Fax: +49 228287 5854; E-mail: astrid.golla@uni-bonn.de

${ }^{5}$ These authors contributed equally to this work.

Received 24 August 2001; revised 24 January 2002; accepted 31 January 2002 adolescents. In general, the disease has a self-limited course, causing few, if any, residual changes. In about a quarter of the patients bony lesions are accompanied by cutaneous manifestations of pustulosis palmoplantaris. ${ }^{1}$ No epidemiological data of CRMO exist and sporadic case reports of affected $\operatorname{sibs}^{2}$ are the only data available concerning a possible familial aggregation. Some observations in our patient sample are in concordance with a causative genetic component of CRMO. We observed two monozygous twin pairs, with both twins affected in one pair and CRMO in one and palmoplantar pustulosis in the second twin in the other pair. Furthermore, there was an affected child whose father showed chronic non-infectious 
osteomyelitis of the sternum, a condition which may be related to or may present a form of CRMO. A possible genetic basis of human CRMO is further suggested by the existence of a highly similar disorder in the mouse (cmo, chronic multifocal osteomyelitis), which results from a spontaneous mutation and follows an autosomal recessive inheritance. The mouse cmo gene was mapped to mouse chromosome 18 by linkage analysis. ${ }^{3}$ Two heritable human disorders are localised on human chromosome 18q, in the region homologous to the cmo mouse localisation: familial expansile osteolysis (FEO) and familial Morbus Paget (Paget disease of bone, PDB). ${ }^{4,5}$ CRMO, FEO and PDB are bone diseases, which share osteoclastic bone resorption resulting in bony lesions as a primary manifestation. A distinct insertion mutation in TNFRSF11A (RANK=receptor activator of nuclear factor $\kappa \mathrm{B}$ ) was shown to cause FEO and another insertion mutation in RANK was detected in one family with PDB. $^{6}$ But in most cases of familial and sporadic Morbus Paget no RANK mutations or variants were detected. ${ }^{7-9}$ Loss of heterocygosity $(\mathrm{LOH})$ analysis in sporadic and Paget associated osteosarcomas revealed a region of minimum $\mathrm{LOH}$ between markers D18S60 and D18S42, suggesting the presence of a PDB and osteosarcoma related tumour suppressor gene in this region. ${ }^{9}$ The identity of the tumor related gene is not clear, but it is probably not identical with RANK, since no mutations in RANK were detected in Paget related osteosarcomas ${ }^{7}$ and since the activating nature of the identified FEO- and PDBmutations in RANK is not compatible with the loss of function type of mutation (suggested by $\mathrm{LOH}$ ) in the assumed PDB osteosarcoma gene. To evaluate the role of the $18 \mathrm{q}$ region in CRMO we conducted a family based association study with markers D18S1148 and D18S60 and a mutation screening in patients with CRMO in RANK and two further genes, chosen because of their location in immediate vicinity to D18S60, Phosphatidylinositol glycan, class N (PIGN, Genbank accession NM_012327) and KIAA1468 (Genbank accession AB040901).

\section{Material and methods Patients}

Twenty-seven patients with CRMO and their parents were collected from several centres in Germany. All patients were from German descent except for one Turkish and one Croatian patient. The diagnosis of CRMO was based on osteolytic lesions with marginal sclerosis in radiography, a good general condition and normal blood count. In addition, there was multifocal and/or characteristic location (metaphysis of long bones or clavicula) and/or accompanying palmoplantar pustulosis. Written informed consent was obtained from all patients and/or their parents. The study protocol was approved by the Ethics Committee of the Medical Department of the University of Munich.

\section{Molecular studies}

The patients and their parents were genotyped using standard methods for the two markers D18S1148 and D18S60 within the FEO and PDB linkage interval on chromosome 18q. D18S60 is immediately adjacent to the minimal region of $\mathrm{LOH}$ in osteosarcoma, the distance between D18S1148 and D18S60 is about $1 \mathrm{Mb}$ physically and $1 \mathrm{cM}$ genetically., ${ }^{9,10}$ Coding sequences of RANK, PIGN (Genbank accession NM_012327) and KIAA1468 (Genbank accession AB040901) from eight affected and two control individuals were amplified in a standard $50 \mu \mathrm{l}$ PCR reaction based on genomic DNA for PIGN and KIAA1468 and based on cDNA in six patients and on genomic DNA in two patients for RANK (in the latter the amplification included the flanking intronic sequences). Thr 5' end of the KIAA1468 gene, which is not given in Genbank, was determined by $5^{\prime}$ RACE experiments and then sequenced in the patients. Analysis of the variant 971G/A in RANK was performed using PCR of exon 9 and BbrPI digestion (the variant A causes the loss of a restriction site), the variant 1692A/T in PIGN was analysed by sequencing exon 15 of PIGN in all 27 patients and their parents. The products were purified on QIAquick columns (Qiagen), sequenced with the Big Dye Terminator chemistry (PE Biosystem) and sequenced on an ABI 377 DNA sequencer. Genescan-500 (TAMRA) (Applied Biosystems) was used as length standard for the polymorphic markers. Primer sequences and detailed conditions are available on request.

\section{Statistical analysis}

The genotype data for markers D18S1148 and D18S60 were statistically analysed by two family based association methods, the haplotype relative risk (HRR) ${ }^{11}$ with Fisher's exact test and the transmission disequilibrium test (TDT) ${ }^{12}$ with $P$-values determined using the binomial distribution with an exact binomial test. $P$-values were compared to a significance level corrected for multiple testing using the Bonferroni correction for two markers with five alleles each, using a correction factor of $5-1=4$ for the number of alleles, as suggested by Schaid, ${ }^{13}$ multiplicated by the number of markers $(2 \times 4=8)(\alpha=0.05: 8=0.00625, \alpha=0.01: 8=0.00125)$. For each marker we tested the four more frequent alleles and combined very rare alleles $(n \leqslant 3)$ in a fifth not tested allele. For further analysis of haplotype data (PIGN Exon 15, 1692A/ T variant and RANK Exon 9, 971G/A variant) Fisher's exact test was applied for association analysis between marker and variants and the TDT as implemented in GENEHUNTER ${ }^{14}$ for association analysis between CRMO and haplotypes.

\section{Results}

No difference of allele frequencies between the transmitted parental alleles (the alleles transmitted to the patients) and the nontransmitted parental alleles (pseudocontrols) was observed for marker D18S1148. A rare allele (166 bp in length) of marker D18S60, described with a frequency of 0.05 
in CEPH pedigrees, was found in heterozygous state in one out of 27 pseudocontrols ( 54 nontransmitted parental alleles) but in 11 out of 27 patients (none of the patients and none of the parents was homozygous for this allele). This difference is highly significant $(P=0.0011<0.00125$, HRR). The D18S60 $166 \mathrm{bp}$ allele was highly preferentially transmitted to the patients. 12 out of the 54 parents were heterozygous for this allele and transmitted the $166 \mathrm{bp}$ allele to the patient 11 times and did not transmit it only once $(P=0.003<0.00625$, TDT). Thus we found a statistically significant association and linkage (in the presence of association) of CRMO with a rare allele (166 bp in length) of marker D18S60. The haplotype relative risk (HRR) for the D18S60 $166 \mathrm{bp}$ allele is 18 (exact 95\% confidence interval 2-798), which illustrates that the gene underlying the observed association contributes to the etiology of CRMO in a significant manner.

Except for published polymorphisms, no mutations were found in the coding regions of RANK. A silent variant in RANK exon 9, 971G $>A$, present in 6 out of 54 transmitted and 2 out of 54 nontransmitted parental alleles, has no significant association with CRMO $(P=0.26)$. Yet it is significantly associated with the D18S60/166 bp allele (RANK/A was present in 5 out of 12 parental chromosomes with the D18S60/166 bp allele and 4 out of 96 without, $P=0.0007)$.

No mutations were found in KIAA1468 as well as in PIGN, with exception of a $\mathrm{A}>\mathrm{T}$ transversion in exon 15 of PIGN $(1692 \mathrm{~A}>\mathrm{T})$. The transversion results in a predicted amino acid exchange of Isoleucin to Leucin, amino acids, which are very similar in hydrophobicity, charge and size, and the transversion was seen twice in homozygous state (in a patient and her unaffected father). This indicates that the transversion is probably a polymorphism rather than a mutation leading to an altered protein function. The PIGN/T variant was present in 14 out of 54 transmitted and 5 out of 54 nontransmitted parental alleles, and associated with CRMO at a statistically not significant level $(P=0.07)$. But there was a very strong association between the D18S60/166 bp allele and the PIGN/T variant with all 12 observed D18S60/166 bp chromosomes carrying the PIGN 1692A $>$ T transversion (and 7 out of 96 not 166 bp chromosomes, $P<0.0001)$ and also between PIGN/T and the RANK/A variant $(P=0.0003)$. The slight association between PIGN and CRMO therefore is probably an expression of the linkage disequilibrium in the region, which is represented by a complete association of the D18S60/166 bp allele with the PIGN 1692A $>$ T variant and less strong association of D18S60/166 bp with the RANK $971 \mathrm{G}>\mathrm{A}$ variant (see Figure 1). This is also seen by application of the TDT to the family data, where CRMO shows the most significant association with the D18S60/ 166 bp allele $(P=0.003)$ and less significant association with the PIGN/T-D18S60/166 bp haplotype $(P=0.02)$, the PIGN/TRANK/A haplotype $(P=0.03)$ and the complete PIGN/TD18S60/166 bp-RANK/A haplotype $(P=0.046)$. The observed linkage disequilibrium is in accordance to the small distance

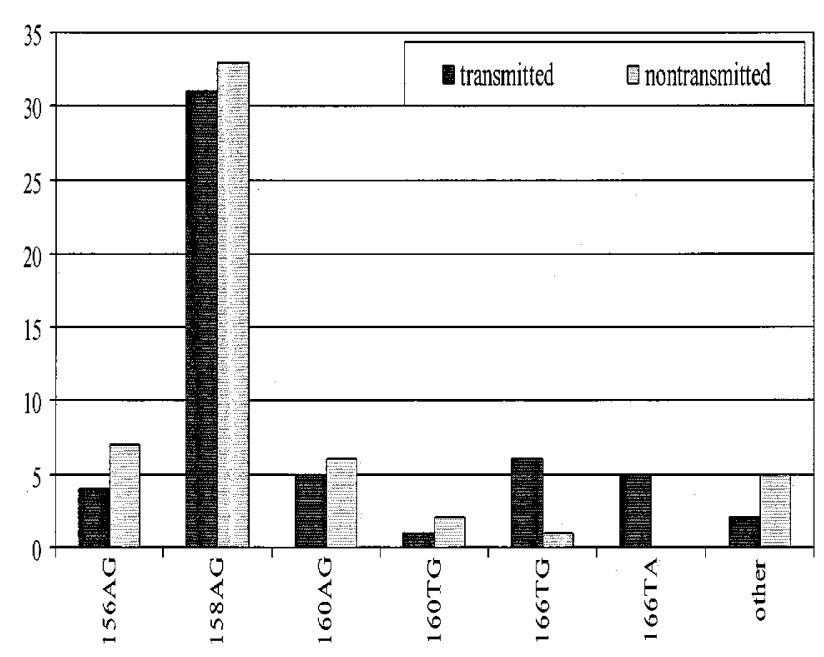

Figure 1 D18S60-PIGN-RANK haplotypes on transmitted and nontransmitted chromosomes of parents of CRMO patients. The length of the D18S60 allele in bp is depicted with the concomitant alleles of PIGN1692 (A or T) and of RANK971 ( $G$ or $A)$, one after the other, haplotypes observed only once or twice are summarised to 'other'.

between D18S60 and PIGN/KIAA1468 (which are immediately adjacent to D18S60) and between D18S60 and RANK, with an estimated distance of approximately $150-200 \mathrm{~kb}$ based on database searches (http://genome.ucsc.edu/).

\section{Discussion}

We found a significant association of CRMO with a rare allele (166 bp) of marker D18S60. The location near D18S60 made TNFRSF11A (RANK=receptor activator of nuclear factor $\kappa \mathrm{B}$ ), the gene mutated in FEO and in one family with $\mathrm{PDB},{ }^{6}$ to a good candidate gene for CRMO. RANK is an osteoclast differentiation and activation factor ${ }^{15}$ and also a regulator of interaction between $\mathrm{T}$ cells and dendritic cells which initiate or regulate immunity. ${ }^{16}$ The similarities between CRMO and FEO/PDB and the clinical picture of inflammatory osteolytic lesions in CRMO supported RANK as a strong candidate for CRMO susceptibility. But by sequencing the coding regions of RANK in a subset of CRMO patients (with and without the susceptibility allele of D18S60) we did not find any mutations in the patients. A silent RANK variant $(971 \mathrm{G}>\mathrm{A})$, observed in half of the patients positive for the rare D18S60 allele, is in linkage disequilibrium with the 166 bp allele of D18S60, but not independently associated with the disease. The same is true for a variant $(1692 \mathrm{~A}>\mathrm{T})$ in the PIGN gene, which was analysed because of its localisation immediately adjacent to D18S60. The linkage disequilibrium confirms the close vicinity of D18S60, PIGN and RANK and is in accordance to the distance of $150-200 \mathrm{~kb}$ between D18S60 and RANK, estimated from database searches. The lack of mutations in RANK in CRMO patients, but also several 
independent facts indicate the existence of another gene near D18S60 involved in bone homeostasis. Except for one RANK mutation found in a single case of familial PDB, so far no mutations or associated variants in RANK were seen in heritable and sporadic cases of PDB. ${ }^{6-8}$ Also, no mutations in RANK were found in PDB associated and sporadic osteosarcomas. ${ }^{6}$ This is in accordance to the obvious contrast of the action in a loss of function manner (LOH) of the assumed tumor suppressor gene located near D18S60 and the gain of function type of the described RANK mutations in FEO and PDB. ${ }^{6}$ Further evidence for a CRMO susceptibility gene different from RANK arises from the phenotype of the recently published RANK knock out mouse. The RANK knock out mouse lacks osteoclasts and has a profound defect in bone resorption and remodelling resulting in osteopetrosis. ${ }^{17}$ The autosomal recessive inheritance of the mouse $\mathrm{cmo}$ phenotype suggests an underlying loss of function mutation. The different phenotypes of the RANK knock out and the cmo mouse suggest that the cmo phenotype does not result from a loss of function mutation of RANK. This observation and the lack of mutations in the patients make an involvement of RANK in CRMO unlikely and point to another gene in this region causing CRMO susceptibility. The genes PIGN and KIAA1468, located immediately adjacent to D18S60, are also very likely not involved in the aetiology of CRMO, as no mutations were found in these genes in CRMO patients. To date, no other genes with a possible functional relevance to CRMO are known in this relatively gene poor region marked by D18S60. A good candidate gene would be the putative tumor suppressor indicated by $\mathrm{LOH}$ in osteosarcomas, ${ }^{9}$ which is not yet identified.

There is not much known about the inheritance pattern of CRMO. Epidemiological data are missing and almost nothing is known about familial aggregation. It is not possible to infer a clear monogenic inheritance pattern from our family sample (27 patients with both parents each and 26 sibs altogether, excluding monozygous twin sibs). Except for the father of one patient and for two monozygous twin sibs all parents and sibs were healthy. A monogenic inheritance (in a dominant as well as a recessive pattern) would only be possible with a reduced penetrance. In CRMO an apparent reduced penetrance is in line with limitation to a distinct age and the intermittent and often mild course. We found a significant association of CRMO with a single allele of marker D18S60 (166 bp in length) in heterozygous state, suggesting a dominant inheritance pattern at a first glance, but a recessive pattern is not excluded. We did not observe a parent-of-origin effect since the $166 \mathrm{bp}$ allele was transmitted from the father five times and from the mother six times. If CRMO is a monogenic disease without heterogeneity, than, based on our observations, the D18S60 $166 \mathrm{bp}$ associated mutation would represent about $40 \%$ of all mutations under an autosomal dominant, and about 30\% under an autosomal recessive model. The observation, that none out of 26 sibs was affected, fits to a dominant model only with a penetrance of at most $30 \%$ (disregarding the parental generation). But it is also compatible with a recessive model with a reduced penetrance of up to $70 \%$, and even the lack of homozygous patients does not render this model unlikely, since for 27 patients only two homozygotes for the D18S60 $166 \mathrm{bp}$ associated mutation would be expected (with the other patients being compound heterozygous for the $166 \mathrm{bp}$ associated and a not associated mutation). So both monogenic inheritance patterns are possible assuming a reduced penetrance, with the recessive one more plausible. But it is also possible, that human CRMO, in contrast to the mouse $\mathrm{cmo}$, is no classical mendelian trait and that the susceptibility gene on 18q21.3-18q22 might lead to CRMO depending on the genetic and/or environmental background. Although the genetic model is not yet clear, the high HRR suggests that the gene underlying the observed association contributes to the etiology of CRMO in a significant manner and concomitanly demonstrates evidence for a genetic basis of CRMO for the first time.

\section{Acknowledgments}

We thank the patients and their families for their participation, $G$ Shen-Ong for helpful discussion and the clinicians for help with patient recruitment: $M$ Borte, Leipzig, $H$ Girschick, Würzburg, $R$ Häfner, Garmisch-Partenkirchen, T Hospach, Stuttgart, V Jansson, München, J and S Killburger, Schalksmühle, RM Küster, Bad Bramstedt, M Mann, St. Augustin, J Meister, Aue, D Meyer, Ulm, W Oelkers and B Riedel, Hamburg, K-H Pulcher, Hargesheim, M Sailer, Innsbruck, C Schauer-Petrowskaja, Berlin-Buch, F Schilling, Mainz, $P$ von Schönaich, Augsburg, P Schütze, Dresden.

\section{References}

1 Carr A, Cole W, Roberton D, Chow C: Chronic multifocal osteomyelitis. J Bone Joint Surg 1993; 75-B: 582-591.

2 Paller AS, Pachmann L, Rich K, Esterly NB, Gonzalez-Crussi F: Pustulosis palmaris et plantaris: Its association with chronic recurrent multifocal osteomyelitis. J Am Acad Dermatol 1985; 12: $927-930$.

3 Byrd L, Grossmann M, Potter M, Shen-Ong GL: Chronic multifocal osteomyelitis, a new recessive mutation on chromosome 18 of the mouse. Genomics 1991; 11: 794-798.

4 Hughes A, Shearman A, Weber J, et al: Genetic linkage of familial expansile osteolysis to chromosome 18q. Hum Mol Genet 1994; 3: $359-361$.

5 Cody JD, Singer FR, Roodman GD, Otterund B, Lewis TB, Leppert M, Leach RJ: Genetic linkage of Paget disease of the bone to chromosome 18q. Am J Hum Genet 1997; 61: $1117-$ 1123.

6 Hughes A, Ralston S, Marken J, et al: Mutations in TNFRSF11A, affecting the signal peptide of RANK, cause familial expansile osteolysis. Nat Genet 2000; 24: $45-48$.

7 Sparks AB, Peterson SN, Bell C, et al: Mutation screening of the TNFRSF11A gene encoding receptor activator of NF kappa B (RANK) in familial and sporadic Paget's disease of bone and osteosarcoma. Calcif Tissue Int 2001; 68: 151-155.

8 Wuyts W, Van Wesenbeeck L, Morales-Piga A, et al: Evaluation of the role of RANK and OPG genes in Paget's disease of bone. Bone 2001; 28: 104-107. 
9 Nellissery MJ, Padalecki SS, Brkanac Z, et al: Evidence for a novel osteosarcoma tumor-suppressor gene in the chromosome 18 region genetically linked with Paget disease of bone. Am J Hum Genet 1998; 63: 817-824.

10 Dib C, Fauré S, Fizames C, et al: A comprehensive genetic map of the human genome based on 5264 microsatellites. Nature 1996; 380: $152-154$

11 Falk C, Rubinstein P: Haplotype relative risks: an easy reliable way to construct a proper control sample for risk calculations. Ann Hum Genet 1987; 51: 227-233.

12 Spielman R, McGinnis R, Ewens W: Transmission test for linkage disequilibrium: the insulin gene region and insulin-dependent diabetes mellitus (IDDM). Am J Hum Genet 1993; 52: 506-516.

13 Schaid DJ: Transmission disequilibrium, family controls, and great expectations. Am J Hum Genet 1998; 63: 935-941.
14 Kruglyak L, Daly MJ, Reeve-Daly MP, Lander ES: Parametric and nonparametric linkage analysis: a unified multipoint approach. Am J Hum Genet 1996; 58: 1347 - 1363.

15 Lacey D, Timms E, Tan H-L, et al: Osteoprotegerin ligand is a cytokine that regulates osteoclast differentiation and activation. Cell 1998; 93: $165-176$.

16 Anderson DM, Maraskovsky E, Billingsley WL, Teepe MC, DuBose RF, Cosman D, Galibert L: A homologue of the TNF receptor and its ligand enhance T-cell growth and dendritic-cell function. Nature 1997; 390: $175-179$.

17 Li J, Sarosi I, Yan X-Q, et al: RANK is the intrinsic hematopoietic cell surface receptor that controls osteoclastogenesis and regulation of bone mass and calcium metabolism. PNAS 2000; 97: $1566-1571$. 